\title{
Falência ovariana precoce
}

\author{
Premature ovarian failure
}

Carmen Regina Leal de Assumpção'

\section{RESUMO}

1 Instituto Estadual de Diabetes e Endocrinologia Luiz Capriglione (IEDE-RJ). Universidade do Estado do Rio de Janeiro (UERJ). Departamento de Endocrinologia Feminina e Andrologia (DEFA) da SBEM. Ambulatório de Obesidade Infanto-Juvenil do IEDE-RJ, Rio de Janeiro, RJ, Brasil
Este artigo irá rever os diversos aspectos da falência ovariana prematura (FOP), definida como o desenvolvimento de hipogonadismo em mulheres antes dos 40 anos, desde a etiopatogenia, com discussões sobre as causas autoimunes, iatrogênicas, ou anormalidades do cromossomo $X$ assim como manifestações clínicas, diagnóstico e tratamento. A maioria das mulheres com essa condição não possui uma história menstrual, específica do desenvolvimento da FOP, mas a infertilidade associada ao diagnóstico é o aspecto mais problemático da doença. Arq Bras Endocrinol Metab. 2014;58(2):132-43

\section{Descritores}

Insuficiência ovariana; falência ovariana precoce; menopausa precoce; infertilidade

\begin{abstract}
This article is a review on different aspects of premature ovarian failure (POF) defined as the development of hypogonadism in women before 40 years of age. The review will discuss the etiopathogeny, autoimmune and iatrogenic causes, abnormalities of chromosome $\mathrm{X}$, as well as clinical manifestations, diagnosis, and treatment. Most of the women with this disorder do not have menstrual history, specific of POF development, but infertility associated with the diagnosis is the most problematic aspect of the disease. Arq Bras Endocrinol Metab. 2014;58(2):132-43
\end{abstract}

Keywords

Ovarian failure; premature ovarian failure; premature menopause; infertility

\section{INTRODUÇÃO}

$\mathrm{M}$ uitas revisões recentes têm discutido o nome apropriado para essa condição, embora muitos pacientes e médicos prefiram o termo menopausa prematura, um termo simples e compreensível que pode ser explicado aos amigos e familiares, mas implica certa sensação de permanência, no entanto, a ovulação e a gravidez podem ocorrer em um momento posterior, por vezes anos após o diagnóstico (1-3).

A falência ovariana precoce (FOP) é definida como falência gonadal antes dos 40 anos de idade. A incidência é de aproximadamente 1 em 250 mulheres com idade de 35 anos e de 1 em 100 com idade de 40 anos. É idiopática em $74 \%$ a $90 \%$ das pacientes. Algumas pacientes com FOP podem produzir estrogênio, ovular, e, em $5 \%$ a $10 \%$ dos casos, conceber e ter uma gravidez e parto sem alterações, assim como ter a função ovariana intermitente (sem amenorreia associada) e menstruação espontânea (embora anor- malidades da função menstrual estejam presentes) (3-9).

Neste artigo, o termo a ser utilizado será falência ovariana precoce (FOP), o qual é adequado e descreve seu diagnóstico.

\section{ETIOLOGIA E PATOGENIA}

Entender a causa da FOP exige uma compreensão do processo de foliculogênese em que muitas das moléculas que regulam a série de eventos na maturação folicular estão envolvidas. $\mathrm{O}$ processo de maturação folicular ocorre de forma contínua e pode demorar até um ano para proceder o início do crescimento de um folículo primordial até se tornar um folículo ovulatório. A granulosa e as células somáticas da teca sintetizam e secretam fatores de crescimento e diferenciação, fatores de transcrição, como o FOXL2, inibina e hormônios esteroides que são regulados pelas 
gonadotrofinas. O resultado da foliculogênese é uma ovulação ou atresia folicular $(2,10-12)$.

A FOP pode ser dividida em primária e secundária. Na primária, ocorrem aberrações genéticas envolvendo o cromossomo X (monossomia, trissomia, translocações, deleções) ou os autossomos. Os mecanismos genéticos estão relacionados a uma redução da dosagem gênica e efeitos não específicos de cromossomos que prejudicam a meiose, diminuindo o pool de folículos primordiais, aumentando a atresia, devido a apoptose ou fracasso de maturação folicular. Os danos nos ovários por autoimunidade, também como causa primária de FOP, são provocados por alteração de subconjuntos de células $T$ e lesão mediada por células $T$, aumento da produção de autoanticorpos de células $\mathrm{B}$, um menor número de linfócitos efetor/citotóxico, diminuindo, assim, o número e a atividade das células Killer. A FOP secundária seria por infecções, ooforectomia bilateral, quimioterapia e radioterapia $(2,5)$ (Figura 1$)$.

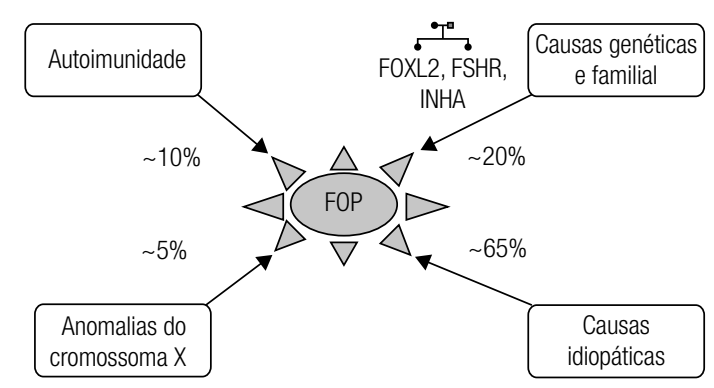

Figura 1. Causas de FOP (2). FOXL2: Forkhead Box L2; FSHR: receptor do FSH; INHA: gene que codifica a subunidade alfa de inibinas A.

Sob a visão folicular, a FOP pode ocorrer por dois mecanismos principais: disfunção e depleção do folículo. A disfunção do folículo indica que, apesar de permanecerem nos ovários, um processo patológico impede sua formação normal $(2,10)$.

A depleção indica que não há folículos primordiais no ovário. Essa condição pode ser devido a uma falha de um conjunto inicial de folículos primordiais, um dispêndio acelerado dos folículos, ou a destruição autoimune ou tóxica destes como alterações no mecanismo que regula a migração das células germinativas, a proliferação mitótica das oogônias e o início da meiose para formar os folículos primordiais $(1,13)$.

Em termos didáticos, podemos classificar a FOP quanto à etiopatogenia em (Tabela 1 ):
Tabela 1. Mecanismos e causas da FOP (1)

\begin{tabular}{|c|c|}
\hline Mecanismo e causa & Comentários \\
\hline \multicolumn{2}{|l|}{ Disfunção do folículo ovariano } \\
\hline \multicolumn{2}{|l|}{ Defeito da sinalização } \\
\hline Mutações do receptor de FSH & $\begin{array}{l}\text { Presença de folículos ovarianos } \\
\text { confirmados por biópsia; doença rara } \\
\text { fora da Finlândia }\end{array}$ \\
\hline $\begin{array}{l}\text { Mutação no receptor do hormônio } \\
\text { luteinizante }\end{array}$ & $\begin{array}{l}\text { Folículos ovarianos presentes no exame } \\
\text { de ultrassom; doença rara }\end{array}$ \\
\hline Mutação da proteína G & $\begin{array}{l}\text { Amenorreia secundária, níveis elevados de } \\
\text { gonadotrofinas e hipoestrogenemia que } \\
\text { respondem à terapia de gonadotrofina; } \\
\text { desenvolvida em paciente com } \\
\text { pseudo-hipoparatireoidismo; doença rara }\end{array}$ \\
\hline \multicolumn{2}{|l|}{ Deficiência enzimática } \\
\hline Deficiência isolada de 17,20-liase & $\begin{array}{l}\text { Folículos ovarianos presentes na biópsia, } \\
\text { aumento do ovário moderado devido ao } \\
\text { bloqueio da síntese de estradiol; doença } \\
\text { rara }\end{array}$ \\
\hline Deficiência de aromatase & $\begin{array}{l}\text { Ovários aumentados ou hiperestimulação } \\
\text { devido à incapacidade do ovário para } \\
\text { aromatizar, androstenediona em estradiol; } \\
\text { doença rara }\end{array}$ \\
\hline \multicolumn{2}{|l|}{ Autoimunidade } \\
\hline Ooforite linfocítica autoimune & $\begin{array}{l}\text { Folículos antrais com infiltração } \\
\text { linfocítica em teca, folículos primordiais } \\
\text { poupados, ovários multifoliculares; } \\
\text { responsável por } 4 \% \text { dos casos de } \\
\text { insuficiência ovariana primária } 46, X X \\
\text { associada a evidências de } \\
\text { autoimunidade adrenal }\end{array}$ \\
\hline \multicolumn{2}{|l|}{ Número insuficiente de folículo } \\
\hline Folículos de Graaf luteinizados & $\begin{array}{l}\text { Folículos antrais visualizados por } \\
\text { ultrassonografia, em } 40 \% \text { das pacientes } \\
\text { com púrpura e insuficiência ovariana } \\
\text { primária espontânea } 46 X X \text {, à base dos } \\
\text { resultados histológicos; } 60 \% \text { dos } \\
\text { folículos antrais fotografados nessas } \\
\text { pacientes eram luteinizados (importante } \\
\text { mecanismo de disfunção folicular nessas } \\
\text { mulheres) }\end{array}$ \\
\hline \multicolumn{2}{|l|}{ Depleção do folículo ovariano } \\
\hline \multicolumn{2}{|l|}{ Número de folículo inicial insuficiente } \\
\hline $\begin{array}{l}\text { Blefarofimose, ptose, síndrome do } \\
\text { epicanto inversus }\end{array}$ & $\begin{array}{l}\text { Mutação em FOXL2 é um mecanismo de } \\
\text { insuficiência ovariana primária familiar; } \\
\text { doença rara }\end{array}$ \\
\hline
\end{tabular}

Perda espontânea acelerada do folículo Síndrome de Turner

Apesar de um complemento normal de folículos primordiais ser estabelecido no ovário durante 0 desenvolvimento fetal, a perda do folículo por meio de apoptose é acelerada; logo o estoque de folículos primordiais está normalmente esgotado antes da puberdade; em oócitos, os dois cromossomos $X$ devem estar presentes $\mathrm{e}$ permanecerem ativos para evitar atresia folicular acelerada; os genes individuais responsáveis pela síndrome nos ovários não foram identificados

Perda do folículo induzido por toxina ambiental

Exposição industrial de Exposição a um solvente de limpeza 2-bromopropano associada à insuficiência ovariana primária (observado em 16 mulheres coreanas) 


\section{Causas genéticas da FOP}

Muitas das famílias estudadas com FOP têm poucos membros, e as análises genéticas são pouco elucidativas, exceto em algumas circunstâncias em que a FOP coexiste com fenótipo que ajuda o rastreio e a identificação da doença (14) (Tabela 2).

\section{Anormalidades cromossômicas X}

Os dois cromossomos $\mathrm{X}$ ativos e intactos são necessários para impedir a atresia folicular acelerada durante a vida pré-natal tardia (após a vigésima semana de gestação). Cerca de $5 \%$ dos casos de FOP são decorrentes de anormalidades no cromossomo $\mathrm{X}$ e envolvem deleções, translocações e alterações numéricas (Tabela 3). A síndrome de Turner é causada por um cariótipo, $45 \mathrm{XO}$ onde um pool normal de folículos é rapidamente perdido antes da puberdade. Nos mosaicismos 45, XO, 45, XO/46, XX, 45, XO/47, XXX, as células germinativas migram para a prega gonadal, mas os oócitos primários sofrem atresia acelerada, provavelmente devido à perda da dosagem diploide de um ou mais genes vitais para o desenvolvimento gonodal (13) (Tabela 3).

Os segmentos cromossômicos FOP l (Xq21.3-q27) e FOP 2 (Xq13.3-q21.1) são importantes para definir a função ovariana. As deleções são localizadas no cromossoma Xq21.3-Xq27, enquanto translocações equilibradas $\mathrm{X} /$ autossômicas foram localizadas no Xq13.3-q21.1. No entanto, nenhum gene envolvido com a FOP foi consistentemente encontrado no cromossoma $\mathrm{X}$ dessas regiões. A exceção é o gene $D I A P H 2$, que foi identificado por pesquisa de microdeleção em um cromossomo X. Também foram observados pacientes com trissomia do 18 e 13. Alguns genes autossômicos têm sido responsabilizados por translocações autossômicas. É interessante notar que um desses possui estreita proximidade com o gene da $\alpha$ inibina (INHA) no cromossoma $2 \mathrm{q} 32.3$, implicado na FOP. Especula-se que essas translocações autossômicas possam ter ocorrido ao acaso e possam aparecer esporadicamente em algumas mulheres com FOP (14-16).

Tabela 2. Associações de síndromes representativas da FOP 46, XX (1)

\begin{tabular}{|c|c|c|c|}
\hline Sindrome & Gene & $\begin{array}{l}\text { Número } \\
\text { OMIM }\end{array}$ & Achados associados importantes \\
\hline Desordens associadas $\mathrm{X}$ frágil & FMR1 ( $X$ frágil, retardo mental 1) & 309550 & $\begin{array}{l}\text { História familiar de deficiência intelectual devido a síndrome } \\
\text { do X frágil ou distúrbio tremor-ataxia }\end{array}$ \\
\hline Síndrome poliendócrina autoimune tipo 1 & AlRE (regulador autoimune) & 240300 & $\begin{array}{l}\text { Insuficiência adrenal, hipoparatireoidismo, candidíase } \\
\text { mucocutânea crônica }\end{array}$ \\
\hline Síndrome poliendócrina autoimune tipo 2 & Desconhecida & 269200 & $\begin{array}{l}\text { Insuficiência adrenal, diabetes melito tipo 1, doença } \\
\text { autoimune da tireoide }\end{array}$ \\
\hline $\begin{array}{l}\text { Hiperplasia adrenal congênita por } \\
\text { deficiência da } 17 \alpha \text {-hidroxilase }\end{array}$ & $\begin{array}{l}\text { CYP17A1 (citocromo P450, família 17, } \\
\text { subfamília A, polipeptídeo 1) }\end{array}$ & 202110 & Hipertensão, alcalose hipocalêmica \\
\hline Deficiência de aromatase & $\begin{array}{l}\text { CYP19A1 (citocromo P450, família 19, } \\
\text { subfamília A, polipeptídeo 1) }\end{array}$ & 107910 & $\begin{array}{l}\text { Virilização materna durante a gravidez, devido à ausência de } \\
\text { aromatase placentária }\end{array}$ \\
\hline $\begin{array}{l}\text { Blefarofimose, ptose, síndrome do } \\
\text { epicanto inversus - BEPS }\end{array}$ & F0XL2 (forkhead box L2) & 110100 & Pálpebras dismórficas \\
\hline $\begin{array}{l}\text { Oftalmoplegia externa progressiva com } \\
\text { deleções do DNA mitocondrial }\end{array}$ & POLG (polimerase [DNA dirigida], gama) & 157640 & $\begin{array}{l}\text { Fraqueza, com início na vida adulta, dos músculos oculares } \\
\text { externos e intolerância ao exercício }\end{array}$ \\
\hline Galactosemia & GALT (galactose-1-fosfato uridil transferase) & 230400 & Hepatomegalia, catarata, deficiência intelectual \\
\hline Doença congênita de glicosilação tipo 1A & PMM2 (fosfomanomutase 2) & 212065 & $\begin{array}{l}\text { Encefalopatia neonatal, hipotonia, retardo psicomotor, } \\
\text { hipoplasia cerebelar, retinite pigmentosa }\end{array}$ \\
\hline Anemia de Fanconi & $\begin{array}{l}\text { FA (grupos de complementação da anemia } \\
\text { de Fanconi) }\end{array}$ & 227650 & $\begin{array}{l}\text { Leucopenia, trombocitopenia, malformações cardíacas, } \\
\text { renais e dos membros, alterações pigmentares da derme }\end{array}$ \\
\hline Ataxia-telangiectasia & ATM (ataxia-telangiectasia mutado) & 208900 & $\begin{array}{l}\text { Ataxia cerebelar, telangiectasias, defeitos do sistema } \\
\text { imunológico, predisposição ao câncer, envelhecimento } \\
\text { precoce, instabilidade do genoma }\end{array}$ \\
\hline Síndrome de Bloom & BLM (síndrome de Bloom) & 210900 & $\begin{array}{l}\text { Envelhecimento precoce, predisposição ao câncer, } \\
\text { instabilidade do genoma }\end{array}$ \\
\hline Síndrome de Werner & WRN (síndrome de Werner) & 277700 & $\begin{array}{l}\text { Envelhecimento precoce, predisposição ao câncer, } \\
\text { instabilidade do genoma }\end{array}$ \\
\hline
\end{tabular}


Tabela 3. Maiores causas de FOP em mulheres (15)

\begin{tabular}{l}
\hline Atresia folicular acelerada \\
\hline Defeitos genéticos \\
Síndrome de Turner \\
Pré-mutações X frágil \\
Deleções e translocações cromossômicas do X \\
Galactosemia \\
Toxinas ovarianas \\
Fármacos quimioterapêuticos (especialmente agentes alquilantes) \\
Caxumba ou infecção por citomegalovírus \\
Radiação \\
Lesão autoimune \\
Isolada ou parte de síndromes poliglandulares autoimunes \\
\hline Estimulação folicular anormal \\
\hline Moduladores intraováricos \\
BMP15, polimorfismos da subunidade alfa-inibina \\
Defeitos enzimáticos esteroidogênicos \\
Deficiência da CPY17 \\
Mutação da StAR \\
Receptores de gonadotrofinas \\
Mutações no receptor de FSH \\
Mutações no gene da subunidade alfa da proteína Gs \\
\hline
\end{tabular}

Pré-mutações FMRl: a síndrome do $\mathrm{X}$ frágil é uma das formas de X-linked mais comuns de retardo mental em todo o mundo. A literatura demonstra uma associação entre FOP e pré-mutações para a síndrome do $\mathrm{X}$ frágil (gene $F M R$, localizado no segmento cromossômico FOPl). As mulheres com a pré-mutação têm risco dez vezes maior de desenvolver FOP. A prevalência de pré-mutação no gene FMRl é de aproximadamente $14 \%$ e, em casos esporádicos, de cerca de $2 \%(17,18)$. O Colégio Americano de Obstetrícia e Ginecologia recomenda que as mulheres com FOP ou um nível elevado de FSH antes da idade de 40 anos, sem causa conhecida, façam rastreamento para premutações FMRl(19).

\section{Causas autossômicas}

Mutações do receptor FSH: o receptor do FSH foi encontrado como um mutante em algumas famílias finlandesas (C566T), levando a FOP. Mutação dos genes da subunidade beta do FSH, do gene $K A L$ e do gene $D A X-1$ altera a síntese e a secreção das gonadotrofinas. A mutação do gene da subunidade beta do FSH foi descrita em uma paciente com amenorreia primária, desenvolvimento precário dos caracteres sexuais secundários e infertilidade. A síndrome de resistência ovariana (ou de Savage) é decorrente de alteração em nível de receptor ou pós-receptor das gonadotrofinas.
A mutação do receptor de $\mathrm{LH}$ pode se manifestar com amenorreia primária ou secundária, sendo que as pacientes descritas atingiram os estádios puberais IV ou V de Tanner $(2,9,13)$.

Galactosemia: é uma doença autossômica recessiva rara causada pela deficiência da enzima galactose-1-fosfato uridil-transferase (GALT), com consequente acúmulo de galactose. Cerca de $80 \%$ das mulheres afetadas desenvolvem FOP, sendo a maioria logo após a puberdade (2).

Mutações no gene BMP15, FOXL2 e NR5A1: os pesquisadores estudam um gene candidato da FOP que pode ser identificado devido a seu papel na função ovariana e/ou desenvolvimento folicular.

Um declínio nos níveis circulantes de inibina, associado a um declínio na reserva folicular, resultou em elevadas concentrações de FSH, maior recrutamento folicular e, consequentemente, aumento da taxa de depleção de folículos. Os padrões hormonais de pacientes com FOP, em comparação às mulheres férteis pareadas por idade, também implicam a inibina como causadora do mecanismo da doença. Portanto, uma mutação no INHA pode ocasionar um aumento na perda de folículo levando a FOP (Figura 2). Aproximadamente 5\% das mulheres com FOP terão uma mutação específica (INHA G769A). Há um suporte funcional para a mutação G769A INHA causando um efeito significativo sobre a função da inibina, embora pareça provável que a mutação não é a única causa genética, mas os fatores ambientais podem também estar envolvidos e, portanto, a mutação pode ser mais um fator de suscetibilidade para o desenvolvimento de FOP $(2,14)$.

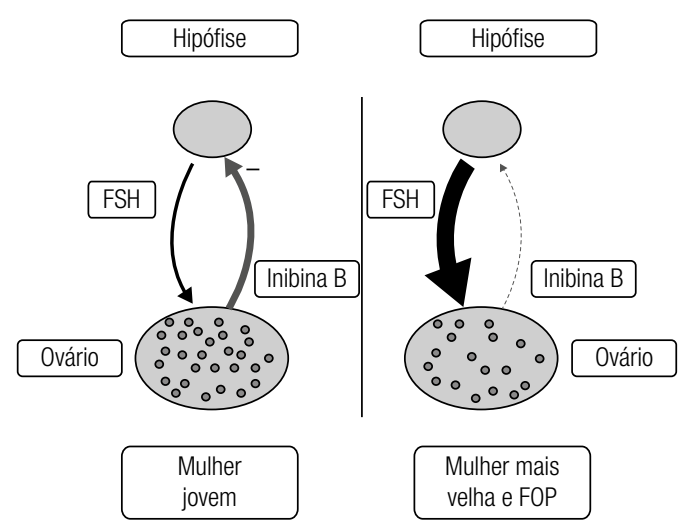

Figura 2. Evidências sugerem que as mulheres mais velhas com diminuição dos níveis de folículos reduziram a produção de inibina $\mathrm{B}$, levando a um aumento da FSH, em comparação com as mulheres jovens. De modo semelhante, há hipótese de que as mulheres com uma mutação no INHA e, por conseguinte, inibina B anormal também cursariam com aumento dos níveis de FSH e aumento das taxas de perda de folículo (2). 


\section{Agentes infecciosos}

Caxumba, infecção por shigella, malária e varicela têm sido implicadas como causas raras de FOP.

\section{Autoimunidade}

Corresponde a cerca de $20 \%$ dos casos de FOP. Ooforite linfocítica, imunoglobulinas bloqueadoras do receptor das gonadotrofinas e anticorpos antigonadotrofinas $(2,13)$.

Associação com outras doenças autoimunes: a FOP está associada à doença de Addison (DA) em $2 \%$ a $10 \%$ dos casos, caracterizando as síndromes poliglandulares autoimunes (SPAs). A FOP está presente em 17\% a $50 \%$ dos casos de SPA tipo 1 e em $4 \%$ a $7 \%$ dos casos de SPA tipo 2. A autoimunidade tireoidiana é a alteração autoimune mais prevalente (14\% a $27 \%$ ) associada à FOP, seguida pela presença de anticorpos anticélulas parietais $(4 \%)$, diabetes melito tipo $1(2 \%)$ e miastenia grave ou positividade para anticorpos antirreceptores de acetilcolina $(2 \%)(13)$.

\section{Quimioterapia, irrradiação e toxinas ambientais}

Inicialmente a quimioterapia destrói as células da granulosa e da teca em proliferação. Posteriormente, os agentes alquilantes promovem alteração do DNA das células dos folículos primordiais não proliferativos (Tabela 4) (15).

A FOP induzida por radiação é dependente da idade da paciente e da dose recebida. Uma dose de radiação ovariana maior ou igual a 600 cGy produz FOP em virtualmente todas as pacientes com mais de 40 anos, mas há diferenças significantes na sensibilidade entre os indivíduos. Com relação às toxinas ambientais, sabe-se que mulheres fumantes apresentam menopausa mais precoce que as não fumantes.

Adultos sobreviventes de câncer infantil foram avaliados quanto a eventos adversos para a saúde endócrina e reprodutiva. A prevalência cumulativa estimada aos 50 anos foi de $86,5 \%$ para a disfunção da hipófise e de 31 ,1\% para FOP (20).

\section{Deficiência enzimática: 17- $\alpha$-hidroxilase, 17,20-liase, colesterol desmolase}

A deficiência de colesterol desmolase (SCC), 17-alfa-hidroxilase $(17 \mathrm{OH}), 17,20$-liase e aromatase pode impedir a síntese de estrógeno, levando a retardo puberal, amenorreia primária e aumento dos níveis de gonadotrofinas, podendo ter folículos primordiais aparentemente normais. A forma clássica de deficiência de SCC não produz nenhum esteroide biologicamente ativo $\mathrm{e}$ as pacientes raramente sobrevivem até a vida adulta. A deficiência da $17 \mathrm{OH}$ leva a hipertensão, hipocalemia, amenorreia primária e ausência do desenvolvimento dos caracteres sexuais secundários. A deficiência da aromatase se manifesta com retardo puberal, amenorreia hipergonadotrófica e múltiplos cistos ovarianos.

A falta de retroalimentação negativa do estrógeno sobre as gonadotrofinas pode resultar em crescimento folicular excessivo, podendo apresentar ovários palpáveis ao exame físico, com risco de torção e infarto ovariano $(9,13)$.

\section{Moduladores intraováricos}

Os defeitos nas gonadotrofinas, em seus receptores ou na proteína $\mathrm{G}$ (sinalização pós-receptor) podem resultar em FOP. Pacientes com pseudo-hipoparatiroidismo têm resistência ovariana, por defeito no sistema de geração de AMP cíclico, amenorreia secundária e hipoestrogenismo hipergonadotrófico (13).

Tabela 4. Toxicidade associada à quimioterapia do ovário (15)

\begin{tabular}{|c|c|}
\hline Droga & Classe (ação) \\
\hline \multicolumn{2}{|c|}{ Definitivamente associada a danos nos ovários } \\
\hline Mecloretamina & Mostarda nitrogenada (agente alquilante) \\
\hline Mostarda L-fenilalanina & Mecloretamina (agente alquilante) \\
\hline Clorambucil & Cloroetilamina (agente alquilante) \\
\hline Ciclofosfamida & Cloroetilamina (agente alquilante) \\
\hline Melfalam & Mecloretamina (agente alquilante) \\
\hline Busulfam & Sulfonato (agente alquilante) \\
\hline Procarbazina & Substituição hidrazina \\
\hline Dacarbazina & Agente alquilante \\
\hline \multicolumn{2}{|c|}{ Provavelmente associada a danos nos ovários } \\
\hline Vinblastina & Alcaloide vinca \\
\hline Citosina-arabinosídeo (Ara-C) & Antimetabólito \\
\hline Cisplatina & Metal pesado \\
\hline Carmustina & Nitrossoureia (agente alquilante) \\
\hline Lomustina & Nitrossoureia (agente alquilante) \\
\hline VP-16 (etoposídeo) & Podofilotoxina \\
\hline Imatinibe & Inibidor da tirosina quinase \\
\hline \multicolumn{2}{|c|}{ Baixa probabilidade de danos nos ovários } \\
\hline Metotrexato & Antimetabólito \\
\hline Fluorouracilo (5-FU) & Antimetabólito \\
\hline 6-mercaptopurina & Antimetabólito \\
\hline Vincristina & Alcaloide vinca \\
\hline Mitomicina & Antibiótico (agente de alquilação) \\
\hline \multicolumn{2}{|l|}{ Desconhecido } \\
\hline Vm-26 & Podofilotoxina \\
\hline Antraciclina & Daunorubicin \\
\hline Bleomicina & Peptídeo \\
\hline Vindesina & Vinca alcaloide \\
\hline Doxorrubicina & Antraciclina \\
\hline
\end{tabular}




\section{Novas abordagens para descobrir genes FOP}

As variantes genéticas podem interagir com outras (epistasia) e com fatores ambientais, tais como dieta, tabagismo, hormônios, exposição a poluentes e outros fatores de estilo de vida. A magnitude do efeito de polimorfismos de nucleotídeos simples (SNP) identificou apenas poder explicar uma pequena proporção do efeito total da genética.

Até o momento, apenas um trabalho significativo sobre o genoma foi realizado em FOP. Os SNP encontrados foram mapeados com um íntron no gene ADAMTS19, que parece desempenhar um papel importante na formação e na função normal das gônadas (2).

\section{DIAGNÓSTICO}

Não há sinais ou sintomas óbvios que antecedem a cessação dos períodos menstruais. A maioria tem idade da menarca, história menstrual e, possivelmente, fertilidade normal antes do aparecimento dos sintomas da FOP. Uma apresentação comum é a falha ao retomar a menstruação após a gravidez ou ao parar de tomar a pílula anticoncepcional oral (2).

Embora o diagnóstico precoce da FOP seja importante para a prevenção da osteoporose e, possivelmente, a prevenção da doença cardíaca coronariana, muitas mulheres têm um atraso significativo no diagnóstico (cerca de cinco anos). Além disso, mais de $50 \%$ das jovens com FOP relataram ter consultado três ou mais clínicos antes de testes de laboratório finais (21).

\section{Diagnóstico diferencial das oligomenorreias e amenorreias}

Na maioria dos casos, a doença desenvolve-se após a puberdade normal e menstruações regulares estabelecidas, embora amenorreia primária possa estar presente em cerca de $10 \%$ dos casos. Mais comumente, existe um pródromo de oligomenorreia, polimenorreia, ou sangramento uterino disfuncional. Apesar de a lista de potenciais causas de amenorreia secundária ser longa, a maioria dos casos é explicada por quatro condições: síndrome dos ovários policísticos (SOP), amenorreia hipotalâmica, hiperprolactinemia e FOP. Aproximadamente $50 \%$ das mulheres com FOP têm função ovariana intermitente, com menstruações imprevisíveis e não de amenorreia completa. Uma definição mais prática seria de quatro meses ou mais de menstruação "desordenadas" em associação com os níveis de FSH da menopausa (1).

Embora se utilizem as dosagens de gonadotrofinas e estradiol para subclassificar as oligo e amenorreias, uma simples dosagem de hormônio antimulleriano (HAM) poderia corresponder à primeira linha de investigação.

Nas mulheres com FOP, os níveis de HAM estão reduzidos e, na amenorreia associada à hiperprolactinemia ou hipogonadismo hipogonadotróficos, níveis de HAM estão normais (22).

\section{Anamnese dirigida}

Uma vez que a gravidez tenha sido descartada, os médicos que avaliam mulheres com amenorreia secundária devem abordar várias questões como: Há algum declínio na saúde geral, como diabetes melito não controlado, doença celíaca e doenças autoimunes (incluindo hipotireoidismo, insuficiência adrenal e hipoparatireoidismo), que pode relacionar a uma síndrome poliglandular autoimune, síndrome de Sjögren, miastenia grave, artrite reumatoide, ou lúpus eritematoso sistêmico? (1).

Há excesso de exercício, ingestão calórica inadequada ou estresse emocional? Há histórico de radioterapia ou quimioterapia prévia? Há galactorreia ou sinais de excesso de andrógenos? Os sintomas da deficiência de estrogênio se desenvolvem em muitas pacientes, como sintomas vasomotores, distúrbios do sono e dispareunia. No entanto, nem todas têm deficiência de estrógeno, e um exame vaginal muitas vezes mostra níveis normais de estrogênio. Uma história familiar da síndrome do X frágil, deficiência mental, demência, tremores ou ataxia ou sintomas semelhantes aos associados à doença de Parkinson pode apontar para uma pré-mutação no gene X frágil (FMRl) (23).

\section{Exame físico}

O exame físico pode revelar indícios de um distúrbio associado, tais como:

Características da síndrome de Turner: baixa estatura, implantação baixa de cabelo, palato em ogiva, peito em escudo com mamilos amplamente espaçados, pescoço alado, quarto e quinto metacarpos curtos.

Ptose palpebral: tem sido associado com uma forma familiar rara de FOP (14).

Bócio: consistente com tiroidite de Hashimoto ou doença de Graves. 
Hiperpigmentação ou vitiligo (associada com insuficiência adrenal autoimune): neste caso, a hipotensão ortostática também pode estar presente (1).

Sinais de vaginite atrófica: embora o exame vaginal normal, incluindo o muco cervical normal e índice de maturação vaginal, também possa ser visto, se tiver havido função ovariana recente (7).

Alargamento do ovário: em função da ooforite linfocítica ou do defeito enzimático esteroidogênico (24).

\section{LABORATÓRIO}

\section{Teste de gravidez}

Hormônios tireoidianos e anticorpos antitireoidianos

Progesterona: a medição de concentrados séricos elevados se associa a amenorreia primária, insuficiência no desenvolvimento puberal, elevação de FSH, deficiências de 17-hidroxilase ou 17-20 desmolase (26).

Prolactina: determinar se a hiperprolactinemia é a causa dos sintomas.

Inibina: especialmente a B.

FSH: determinar se a paciente é hipergonadotrófica. Em casos de amenorreia causada por estresse (isto é, amenorreia hipotalâmica), o nível de FSH estará na faixa baixa ou normal. Se o nível de FSH estiver no intervalo de menopausa, tal como definido pelo laboratório de referência, o teste deverá ser repetido ao longo de um mês com uma medição de estradiol no soro (1).

Estradiol: a experiência mostra que, dependendo do ensaio, a baixa de estradiol, em particular, não é tão confiável como a avaliação de FSH para identificar a FOP (7).

\section{Autoanticorpos circulantes}

Anticorpos antiovários: são encontrados em até $67 \%$ das pacientes com FOP isolada, em $75 \%$ daquelas com FOP associada a outras doenças autoimunes e em 78\% com cirurgia pélvica (9). Pode ser um epifenômeno secundário à liberação de antígenos após o dano celular. Eles estão presentes na fase inicial da destruição folicular, mas desaparecem em estágios mais tardios (13). O valor preditivo do teste disponível no mercado é pobre como um método para identificar as mulheres com FOP. Num estudo de 26 mulheres com FOP e 26 mulheres com ciclos normais, $50 \%$ das mulheres com FOP e $31 \%$ de mulheres normais tinham anticorpos antiovarianos (27).
Anticorpos antirreceptores de gonadotrofinas: são bloqueadores do receptor de $\mathrm{FSH}$, identificados em três pacientes com mistenia grave e hipogonadismo hipergonadotrófico, sugestivo de mecanismo imunológico comum. Cerca de $80 \%$ das pacientes com FOP tinham imunoglobulinas (IgGs) que bloqueavam a síntese de DNA induzida por FSH nas células da granulosa, indicando interferência com a função pós-receptor. Esses estudos utilizaram receptores de gonadotrofinas não humanos $(9,13)$.

Anticorpos antizona pelúcida: interferem com a interação espermatozoide-oócito e com o desenvolvimento folicular. Presentes em 5,6\% das mulheres com infertilidade, $9 \%$ daquelas com FOP e em $50 \%$ das pacientes menopausadas, sugerindo que a reatividade contra proteínas ovarianas possa ser secundária ao dano folicular, mais que uma resposta autoimune primária.

Anticorpos anticélulas produtoras de esteroides: são IgGs que se ligam às células produtoras de esteroides (ACPE) dos ovários (células de teca, hilares, da granulosa e corpo lúteo), testículos (células de Leydig), placenta (sinciciotrofoblasto) e córtex adrenal. Presentes em $87 \%$ das mulheres com FOP associada à doença de Addison (DA), mas raramente encontrados (2\% a $7 \%$ ) na ausência de DA. Uma vez que $40 \%$ das pacientes com DA e função ovariana normal, positivas para ACPE, desenvolvem falência gonodal após um período de 10 a 15 anos da detecção desses anticorpos, eles são considerados marcadores de FOP (9).

Os ACPE podem ser citotóxicos para as células de granulosa humanas em cultura. São reativos predominantemente contra a SCC, $17 \mathrm{OH}$, e a $3 \beta$-hidroxiesteroide

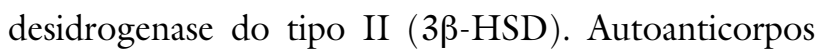
anti-3 $\beta$-HSD, detectados por "immunoblot", foram encontrados em $21 \%$ das pacientes com FOP, enquanto os dosados por meio de imunoprecipitação, em apenas $2 \%$ a $12 \%$ dos casos de FOP, um baixo significado como marcador diagnóstico de FOP $(9,13)$.

Anticorpos suprarrenais: são positivos (4\%), com o uso de imunofluorescência indireta (23).

\section{HAM}

É uma glicoproteína produzida pelas células granulosas de folículos ovarianos primários, pré-antrais e pequenos folículos antrais. Pode ser aplicável na individualização do risco de injúria gonadal iatrogênica em mulheres portadoras de neoplasia que serão submetidas à quimioterapia e na predição da idade de menopausa e do prognós- 
tico reprodutivo da mulher, fornecendo bases sólidas ao aconselhamento conceptivo e contraceptivo (22).

\section{Genética: cariótipo, cromossomas e genes}

$\mathrm{O}$ risco de ter um cariótipo anormal é tanto maior quanto menor a idade de instalação da FOP. Um cariótipo deve ser realizado como parte da avaliação de base para todos os doentes com FOP, com ou sem os estigmas da síndrome de Turner. A síndrome de Turner, monossomia do X, afeta um por 2.500 mulheres no nascimento. As principais características são baixa estatura e FOP. A baixa estatura e monossomia de um cromossoma X maternos têm sido implicados em incapacidades na vida adulta (29).

Mulheres com anormalidades cromossômicas X deram luz a crianças normais que, posteriormente, desenvolveram FOP. Embora raro em mulheres com FOP, aquelas com material presente de cromossoma $\mathrm{Y}$ exigem ooforectomia por causa do risco de tumores (30).

$\mathrm{O}$ gene FMRl está no cromossoma Xq27 do segmento cromossômico FOPl. Possuindo menos que 50 repetições, é considerado normal, enquanto com mais de 200 repetições é considerado mutação completa, e os homens portadores podem desenvolver a síndrome X frágil. As expansões entre 45 e 200 repetições de uma repetição CGG no 5'-UTR do gene são conhecidas como pré-mutações (18).

O Colégio Americano de Obstetrícia e Ginecologia recomenda que as mulheres com FOP ou um nível elevado de FSH antes da idade de 40 anos, sem causa conhecida, façam rastreamento para pré-mutações FMRl (19).

\section{Imagem}

Ultrassonografia pélvica: identifica casos envolvendo ovários aumentados com múltiplos folículos que podem ser submetidos à torção, como na deficiência isolada de 17,20-liase. Na ooforite autoimune pode apresentar aspecto normal apesar da FOP (Figura 3) (33).

Densidade mineral óssea: a avaliação da densidade mineral óssea com a DXA deve ser feita, pois a FOP ocorre antes do período natural da menopausa. Mulheres jovens poderão ter sua massa óssea comprometida (35).

\section{Biópsia}

A biópsia do ovário não fornece informação útil ao tratamento da FOP e, portanto, não é indicada; gravidez pode ocorrer mesmo após a biópsia mostrar que

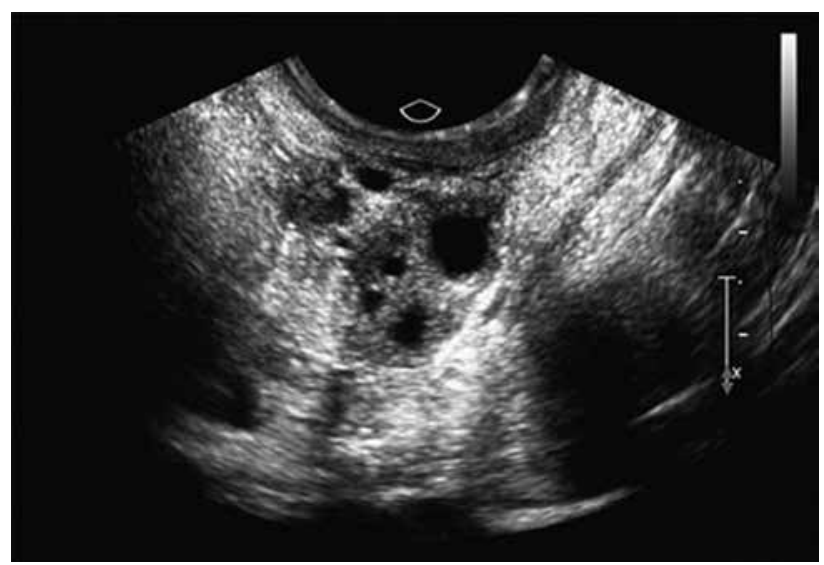

Figura 3. Ecografia transvaginal mostrando ovário de aspecto normal, com a presença de múltiplos folículos. Ooforite com infiltração tecal por linfócitos, histologicamente confirmada por meio de uma biópsia ovariana realizada quando a paciente tinha 26 anos de idade (1).

os folículos estão ausentes. A evidência histológica de ooforite está presente em $11 \%$ das pacientes com FOP. Todas as pacientes positivas para anticorpos anticélulas produtoras de esteroides têm ooforite linfocítica, mas é um achado excepcional (menos de 3\%) nas pacientes com FOP isolada ou não associada à doença autoimune $(1,9,13,34)$.

Apesar de algumas evidências, a ultrassonografia pélvica e a biópsia dos ovários não têm nenhum benefício clínico comprovado no manejo da FOP. Como exemplo, a biópsia do ovário é imprecisa devido a erro de amostragem, e a gravidez tem sido relatada em mulheres que se submeteram à biópsia aberta em cunha e que não apresentaram folículos (34).

\section{TRATAMENTO}

\section{Terapia hormonal (TH)}

A reposição hormonal para mulheres jovens com FOP deve imitar a função ovariana normal, tanto quanto possível. Muitas mulheres com FOP se beneficiariam do alívio sintomático por meio da utilização de esteroides exógenos, para compensar a perda do estradiol (E2) e, possivelmente, progesterona e androgênios. Os sintomas da menopausa, como fogachos, suores noturnos e secura vaginal, podem ser aliviados com a reposição de estrogênio. As mulheres que estão preocupadas em evitar a gravidez muitas vezes são aconselhadas a tomar a pílula anticoncepcional oral combinada. Em mulheres com útero intacto, o estrogênio deverá ser administrado em combinação com uma progestina para evitar a hiperplasia do endométrio (2). 


\section{Tipo de terapia com estrogênio}

A supressão de concentrações de gonadotrofinas endógenas com doses farmacológicas de estrogênio anteriores à terapia de gonadotropinas foi avaliada para melhorar as taxas de ovulação em cerca de alguns, mas não todos os estudos (32).

Para controlar os sintomas vasomotores e estrogenizar totalmente o epitélio vaginal, a maioria das mulheres com FOP inicia doses de substituição completa de estrogênios, tais como E2 transdérmico (geralmente $100 \mathrm{mcg}$ por dia) ou estradiol por via oral (geralmente de $2 \mathrm{mg} /$ dia) $(1,37)$.

Para as meninas que ainda não completaram a maturação óssea com amenorreia primária, nas quais as características sexuais secundárias não se desenvolveram inicialmente, devem ser dadas doses muito baixas de estrogênio na tentativa de imitar a maturação puberal gradual, com a monitorização da idade óssea e da progressão puberal quando, então, se introduz a progesterona $(38,39)$.

\section{Progestógenos}

Como a maioria das pacientes possui útero intacto, um regime de progestina é fundamental para reduzir completamente o risco de hiperplasia e carcinoma do endométrio por um período de 12 dias ao mês $(1,40)$

\section{Anticoncepcionais}

Uma vez que a atividade ovariana espontânea pode continuar, a paciente que opta por um contraceptivo oral deve ser orientada sobre a importância do cumprimento e da possibilidade de que os sintomas vasomotores podem retornar durante o intervalo de quatro a sete dias livre de pílula $(1,3,41,42)$.

Quando a terapia hormonal de estrogênio e progesterona é prescrita, um regime cíclico irá induzir regularmente a menstruação permitindo e facilitando o potencial de $5 \%$ a $10 \%$ para uma gravidez espontânea e inesperada. Essas gravidezes espontâneas progridem inteiramente normais na maioria dos casos e não parece haver necessidade de suplementação hormonal exógena durante a gravidez (6).

\section{Duração da terapia com estrogênio}

A maioria das pacientes está ciente da associação entre a deficiência de estrogênio e a osteoporose, mas elas também estão preocupadas com os potenciais riscos de complicações cardiovasculares e câncer de mama com a terapia estrogênica de longo prazo, que têm sido relatados em mulheres pós-menopáusicas mais velhas (37).

No estudo da Women's Health Initiative (WHI), a menopausa precoce tem sido associada a um aumento da incidência de fraturas, aumento da mortalidade total e mortalidade por doença isquêmica $(36,37)$. Em mulheres jovens com FOP, os benefícios da terapia de reposição hormonal geralmente superam os riscos potenciais (23). A ausência de reposição de estrogênio nas mulheres jovens pode ocasionar maior risco de doença cardíaca coronária tardia, possivelmente devido à deficiência de estrogênio (44-47). Como um exemplo, dois estudos sugerem que as mulheres com FOP apresentam disfunção endotelial vascular significativa, a qual é restaurada ao normal por terapia com estrogênio $(45,46)$. A maioria dos especialistas concorda que as mulheres jovens com FOP devem substituir os hormônios do ovário, normalmente, pelo menos até a idade de 50 anos (48).

\section{Deficiência androgênica}

É controverso se as mulheres com POF também devem fazer a suplementação androgênica, no entanto, revisões recentes têm apoiado a adição de testosterona à TH para melhorar a função sexual e o bem- estar (46). Os potenciais efeitos colaterais da reposição androgênica incluem hirsutismo e acne. Contudo, nas mulheres com insuficiência adrenal coexistente, a terapia de androgênio com de-hidroepiandrosterona parece ser benéfica (50-52).

\section{Manutenção da saúde óssea}

Em um pequeno estudo randomizado, os contraceptivos orais suprimiram os marcadores de formação óssea em comparação com a substituição mais fisiológica por esteroides sexuais em mulheres jovens com FOP. Contudo, a densidade mineral óssea (DMO) da coluna lombar melhorou em ambos os grupos. É incerto se a administração de doses fisiológicas de estrogênio seguida por uma progestina é melhor para a saúde do esqueleto do que doses farmacológicas de um regime de estrogênio-progestina combinado (ou seja, os contraceptivos orais) (43).

Se a mulher optar por não tomar suplementação de estrogênio, então a DMO precisa ser monitorada de perto, e o uso de bifosfonatos ou outros medicamentos deve ser considerado $(1,49)$. Não existem da- 
dos disponíveis especificamente para essas mulheres no que diz respeito à ingestão diária recomendada de cálcio e de vitamina $\mathrm{D}$ e a frequência e intensidade de exercício de peso recomendado, com ingestão de $1.200 \mathrm{mg}$ de cálcio elementar por dia, e manutenção adequada de vitamina $\mathrm{D}$, que é definida como uma concentração de nível 25- hidroxivitamina $\mathrm{D}$ de 30 ng por mililitro (75 nmol por litro) ou mais alta. A vitamina $\mathrm{D}$ é recomendada com a exposição adequada ao sol e ingesta de pelo menos 800 a 1.000 UI de vitamina D3 por dia. As mulheres devem ser encorajadas a se envolver em uma série de exercícios, como corrida, caminhada, juntamente com exercícios de resistência, e evitar fatores de risco como o tabagismo e o consumo elevado de álcool $(1,40,49)$. Os bisfosfonatos não são aconselhados se a gravidez é possível, uma vez que esses agentes têm uma longa meia-vida no esqueleto e os efeitos sobre o feto são incertos (1).

\section{Infertilidade}

A infertilidade é um problema significativo para a maioria das mulheres, no entanto, a ovulação e a gravidez bem-sucedida podem ocorrer em cerca de $5 \%$ a $10 \%$ da pacientes $(53)$.

\section{Restauração da fertilidade}

Terapia gonadotrópica: a terapia da indução da ovulação com gonadotrofinas é muitas vezes tentada, mas as taxas de ovulação e gravidez são baixas. As gonadotrofinas exógenas teoricamente poderiam exacerbar a FOP autoimune não reconhecida $(5,6,8)$.

Combinado com estrogênio: tem-se pensado que o estrogênio exógeno pode ter um efeito benéfico sobre a ovulação e fertilidade, com base na observação de que alguns relatos de gravidez na FOP ocorreram nas mulheres que tomam estrogênio (26). A supressão de concentrações de gonadotrofinas endógenas com doses farmacológicas de estrogênio anteriores à terapia de gonadotrofinas foi avaliada, uma vez que o estrogênio pode ter um efeito saudável na gênese do folículo e concepção, melhorando as taxas de ovulação em alguns casos (32). Em um estudo randomizado, controlado com placebo, o tratamento com $150 \mathrm{mcg}$ de etinilestradiol/dia durante duas semanas, antes e durante a estimulação com FSH recombinante, levou às taxas ovulatórias significativamente maiores no grupo de estrogênio (32\%) quando comparado com o grupo placebo $(0 \%)$. A ovulação ocorreu somente em mulheres cujas concentrações séricas de FSH foram suprimidas para $\leq 15$ UI/L com estrogênio (28).

Com agonista de GnRH: a supressão de concentrações de gonadotrofinas endógenas com um agonista de $\mathrm{GnRH}$, antes da terapia de gonadotrofina, não parece melhorar as taxas de ovulação (32).

Terapia com glicocorticoides: a terapia com glicocorticoides para o tratamento de suspeita de FOP autoimune também não foi aprovada e pode acarretar o risco de síndrome de Cushing iatrogênica e osteonecrose do quadril (54).

Doação de óvulos: as mulheres com FOP são potenciais candidatas à fertilização in vitro (FIV) com óvulos de doadoras. Em um relatório de $61 \mathrm{mu}$ lheres com FOP, 90 se submeteram ao tratamento cíclico e a chance cumulativa de gravidez após três ciclos foi de aproximadamente $90 \%$. As taxas de sucesso para esse processo dependerão principalmente da idade da dadora do oócito. Esse procedimento não é garantido em ser bem-sucedido, com uma taxa de nascidos vivos de $\sim 30 \%$ por embrião transferido $(31,53)$.

Outro aspecto importante desse processo é que o bebê resultante da fertilização não terá qualquer material genético das mulheres com FOP (53). As mulheres com FOP que engravidam como resultado da doação de oócitos podem ter um risco aumentado de crianças pequenas para a idade gestacional e hipertensão induzida pela gravidez e hemorragia pós-parto, mas esses resultados são controversos (1).

Criopreservação: a criopreservação de tecido ovariano ou oócitos para mais tarde, em crescimento e maturação in vitro, pode ser possível, no entanto, como as mulheres apresentam sintomas da FOP, provavelmente terão folículos que serão de qualidade inferior, o que exigiria que só as mulheres que estão conscientes do futuro da FOP iminente fossem capazes de usar essa tecnologia. Atualmente, a maturação in vitro de folículos imaturos é possível, mas o crescimento, a maturação e a armazenagem in vitro de tecidos ovarianos não são possíveis em humanos. Transplante de ovário tem sido realizado em casos raros em que o paciente tem um gêmeo idêntico com função ovariana normal (53).

Apoio psicossocial: os sentimentos de dor intensa e perda para os filhos biológicos que esperavam ter são normais para os casais cujos planos de vida incluíram a construção de uma família (1). 


\section{CONCLUSÕES}

As mulheres com FOP devem ser encorajadas a manter um estilo de vida que otimize a saúde óssea e cardiovascular, com uma dieta saudável para evitar a obesidade e com tratamento de eventuais fatores de risco identificados (1).

Declaração: a autora declara não haver conflitos de interesse científico neste estudo.

\section{REFERÊNCIAS}

1. Nelson LM. Primary ovarian insufficiency. N Engl J Med. 2009;360(6):606-14.

2. Shelling AN. Premature ovarian failure. Reproduction. 2010;140(5):633-41.

3. Panay N, Kalu E. Management of premature ovarian failure. Best Pract Res Clin Obstet Gynaecol. 2009;23:129.

4. Coulam CB, Adamson SC, Annegers JF. Incidence of premature ovarian failure. Obstet Gynecol. 1986;67:604-6.

5. Vujović S, Ivović M, Tancić-Gajić M, Marina L, Barać M, Arizanović $Z$, et al. Premature ovarian failure. Srp Arh Celok Lek. 2012;140(1112):806-11.

6. van Kasteren YM, Schoemaker J. Premature ovarian failure: a systematic review on therapeutic interventions to restore ovarian function and achieve pregnancy. Hum Reprod Update. 1999;5:483-92.

7. Rebar RW, Connolly HV. Clinical features of young women with hypergonadotropic amenorrhea. Fertil Steril. 1990; 53:804-10.

8. Taylor AE, Adams JM, Mulder JE, Martin KA, Sluss PM, Crowley WF Jr. A randomized, controlled trial of estradiol replacement therapy in women with hypergonadotropic amenorrhea. J Clin Endocrinol Metab. 1996;81:3615-21.

9. Pardini DP, Siva RC, Clapauch R (org). Sociedade Brasileira de Endocrinologia e Metabologia. Associação Médica Brasileira e Conselho Federal de Medicina. Projeto Diretrizes. Falência ovariana precoce, 2006.

10. Picton HM, Harris SE, MuruvoiW, Chambers EL. The in vitro growth and maturation of follicles. Reproduction. 2008;136:703-15.

11. Harris SE, Chand AL, Gersak K, Aittomaki K, Winship I, Shelling AN. Identification of novel mutations in FOXL2 associated with premature ovarian failure. Mol Hum Reprod. 2002;8:729-33.

12. Shah SP, Köbel M, Senz J, Morin RD, Clarke BA, Wiegand KC, et al. Mutation of FOXL2 in granulosa-cell tumors of the ovary. $\mathrm{N}$ Engl J Med. 2009;360(26):2719-29.

13. Falência ovariana precoce. Ruth Clapauch (org.). In: Pardini DP. Endocrinologia feminina e andrologia: manual prático para endocrinologistas, ginecologistas, urologista e médicos com interesse na área. São Paulo: A.C. Farmacêutica; 2012. p. 361-74.

14. Crisponi L, Deiana M, Loi A, Chiappe F, Uda M, Amati $P$, et al. The putative forkhead transcription factor FOXL2 is mutated in blepharophimosis/ptosis/epicanthus inversus syndrome. Nat Genet. 2001;27:159-66.

15. Nelson LM. Pathogenesis and causes of spontaneous primary ovarian insufficiency (premature ovarian failure) UpToDate. 2013. Disponivel em: <http://www.uptodate.com/online>. Acesso em: Jun 18, 2013.

16. Bione S, Sala C, Manzini C, Arrigo G, Zuffardi O, Banfi S, et al. A human homologue of the Drosophila melanogaster diaphanous gene is disrupted in a patient with premature ovarian failure: evidence for conserved function in oogenesis and implications for human sterility. Am J Hum Genet. 1998;62:533-41.

17. Wittenberger MD, Hagerman RJ, Sherman SL, McConkieRosell A, Welt CK, Rebar RW, et al. The FMR1 premutation and reproduction. Fertil Steril. 2007;87:456-65.

18. Sherman SL. Premature ovarian failure in the fragile $X$ syndrome. Am J Med Genet. 2000;97:189-94.

19. American College of Obstetricians and Gynecologists Committee on Genetics. ACOG committee opinion. No. 338: Screening for fragile X syndrome. Obstet Gynecol. 2006;107:1483-5.

20. Hudson MM, Ness KK, Gurney JG, Mulrooney DA, Chemaitilly W, Krull KR, et al. Clinical ascertainment of health outcomes among adults treated for childhood cancer. JAMA. 2013;309(22):2371-81. Erratum in: JAMA. 2013 Jul 3;310(1):99.

21. Alzubaidi NH, Chapin HL, Vanderhoof VH, Calis KA, Nelson LM. Meeting the needs of young women with secondary amenorrhea and spontaneous premature ovarian failure. Obstet Gynecol. 2002;99:720-5.

22. Romão GS, Navarro PA. Clinical use for anti-mullerian hormone in gynecology. Rev Bras Ginecol Obstet. 2013;35(3):136-40.

23. Mayo clinic. Disponível em: http://www.mayoclinic.com/health/ premature-ovarian-failure/DS00843. Acesso em: 5 set, 2013.

24. Biscotti CV, Hart WR, Lucas JG. Cystic ovarian enlargement resulting from autoimmune oophoritis. Obstet Gynecol. 1989;74:492-5.

25. Fernandes AM, Arruda Mde S, Bedone AJ. Twin gestation two years after the diagnosis of premature ovarian failure in a woman on hormone replacement therapy. A case report. J Reprod Med. 2002;47:504-6.

26. Pellicer A, Miró F, Sampaio M, Gómez E, Bonilla-Musoles FM. In vitro fertilization as a diagnostic and therapeutic tool in a patient with partial 17,20-desmolase deficiency. Fertil Steril. 1991;55:970-5.

27. Novosad JA, Kalantaridou SN, Tong ZB, Nelson LM. Ovarian antibodies as detected by indirect immunofluorescence are unreliable in the diagnosis of autoimmune premature ovarian failure: a controlled evaluation. BMC Womens Health. 2003;3:2.

28. Tartagni M, Cicinelli E, De Pergola G, De Salvia MA, Lavopa C, Loverro G. Effects of pretreatment with estrogens on ovarian stimulation with gonadotropins in women with premature ovarian failure: a randomized, placebo-controlled trial. Fertil Steril. 2007;87:858-61.

29. Gould HN, Bakalov VK, Tankersley C, Bondy CA. High levels of education and employment among women with Turner syndrome. J Womens Health (Larchmt). 2013;22(3):230-5.

30. Gemmill RM, Pearce-Birge L, Bixenman H, Hecht BK, Allanson JE. Y chromosome--specific DNA sequences in Turner-syndrome mosaicism. Am J Hum Genet. 1987;41:157-67.

31. Paulson RJ, Hatch IE, Lobo RA, Sauer MV. Cumulative conception and live birth rates after oocyte donation: implications regarding endometrial receptivity. Hum Reprod. 1997;12:835-9.

32. Surrey ES, Cedars MI. The effect of gonadotropin suppression on the induction of ovulation in premature ovarian failure patients. Fertil Steril. 1989;52:36-41.

33. Bakalov VK, Anasti JN, Calis KA, Vanderhoof VH, Premkumar $A$, Chen $S$, et al. Autoimmune oophoritis as a mechanism of follicular dys-function in women with $46, X X$ spontaneous premature ovarian failure. Fertil Steril. 2005;84:958-65.

34. Khastgir G, Abdalla H, Studd JW. The case against ovarian biopsy for the diagnosis of premature menopause. Br J Obstet Gynaecol. 1994;101:96-8.

35. Anasti JN, Kalantaridou SN, Kimzey LM, Defensor RA, Nelson LM. Bone loss in young women with karyotypically normal spontaneous premature ovarian failure. Obstet Gynecol. 1998;91:12-5. 
36. Rossouw JE, Anderson GL, Prentice RL, LaCroix AZ, Kooperberg C, Stefanick ML, et al.; Writing Group for the Women's Health Initiative Investigators. Risks and benefits of estrogen plus progestin in healthy postmenopausal women: principal results From the Women's Health Initiative randomized controlled trial. JAMA. 2002;288(3):321-33.

37. Mishell DR Jr, Nakamura RM, Crosignani PG, Stone S, Kharma K, Nagata $Y$, et al. Serum gonadotropin and steroid patterns during the normal menstrual cycle. Am J Obstet Gynecol. 1971;111:60-5.

38. Canonico M, Oger E, Plu-Bureau G, Conard J, Meyer G, Lévesque H, et al.; Estrogen and Thromboembolism Risk (ESTHER) Study Group. Hormone therapy and venous thromboembolism among postmenopausal women: impact of the route of estrogen administration and progestogens: the ESTHER study. Circulation. 2007;115:840-5.

39. Cirillo DJ, Wallace RB, Rodabough RJ, Greenland P, LaCroix $A Z$, Limacher MC, et al. Effect of estrogen therapy on gallbladder disease. JAMA. 2005;293:330-9.

40. The Post-menopausal Estrogen/Progestin Interventions (PEPI) Trial. Effects of hormone replacement therapy on endometrial histology in postmenopausal women. The Writing Group for the PEPITrial. JAMA. 1996;275:370-5.

41. Alper MM, Jolly EE, Garner PR. Pregnancies after premature ovarian failure. Obstet Gynecol. 1986;67:59S-62S.

42. Kaunitz AM. Clinical practice. Hormonal contraception in women of older reproductive age. N Engl J Med. 2008;358:1262-70.

43. Crofton PM, Evans N, Bath LE, Warner P, Whitehead TJ, Critchley $\mathrm{HO}$, et al. Physiological versus standard sex steroid replacement in young women with premature ovarian failure: effects on bone mass acquisition and turnover. Clin Endocrinol (Oxf). 2010;73:707-14.

44. Atsma F, Bartelink ML, Grobbee DE, van der Schouw YT. Postmenopausal status and early menopause as independent risk factors for cardiovascular disease: a meta-analysis. Menopause. 2006;13:265-79.
45. Kalantaridou SN, Naka KK, Papanikolaou E, Kazakos N, Kravariti $M$, Calis $K A$, et al. Impaired endothelial function in young women with premature ovarian failure: normalization with hormone therapy. J Clin Endocrinol Metab. 2004;89:3907-13.

46. Mondul AM, Rodriguez C, Jacobs EJ, Calle EE. Age at natural menopause and cause-specific mortality. Am J Epidemiol. 2005;162:1089-97.

47. Ostberg JE, Storry C, Donald AE, Attar MJ, Halcox JP, Conway GS. A dose-response study of hormone replacement in young hypogonadal women: effects on intima media thickness and metabolism. Clin Endocrinol (Oxf). 2007;66:557-64.

48. Kalantaridou SN, Nelson LM. Premature ovarian failure is not premature menopause. Ann NY Acad Sci. 2000;900:393-402.

49. Drillich $A$, Davis SR. Androgen therapy in women: what we think we know. Exp Geront. 2007;42:457-62.

50. Bernardi F, Hartmann B, Casarosa E, Luisi S, Stomati M, Fadalti $M$, et al. High levels of serum allopregnanolone in women with premature ovarian failure. Gynecol Endocrinol. 1998;12:339-45.

51. Bachelot A, Meduri G, Massin N, Misrahi M, Kuttenn F, Touraine P. Ovarian steroidogenesis and serum androgen levels in patients with premature ovarian failure. J Clin Endocrinol Metab. 2005;90:2391-6.

52. Elias AN, Pandian MR, Rojas FJ. Serum levels of androstenedione, testosterone and dehydroepiandrosterone sulfate in patients with premature ovarian failure to age-matched menstruating controls. Gynecol Obstet Invest. 1997;43:47-8.

53. Pérez-Andújar A, Newhauser WD, Taddei PJ, Mahajan A, Howell $\mathrm{RM}$. The predicted relative risk of premature ovarian failure for three radiotherapy modalities in a girl receiving craniospinal irradiation. Phys Med Biol. 2013;58(10):3107-23.

54. Kalantaridou SN, Braddock DT, Patronas NJ, Nelson LM. Treatment of autoimmune premature ovarian failure. Hum Reprod. 1999;14:1777-82. 